Modelo de capital intelectual para la investigación en las universidades públicas de la Costa Caribe colombiana

Intellectual capital model for research in public universities in the colombian Caribbean Coast

\author{
Volumen 17, Número 1 \\ Enero-Abril \\ pp. 1-27
}

Este número se publicó el $1^{\circ}$ de enero de 2017

DOI: http://dx.doi.org/10.15517/aie.v17i1.27193

Carlos Ramón Vidal Tovar

Revista indizada en REDALYC, $\underline{\text { SCIELO }}$

Revista distribuida en las bases de datos:

LATINDEX, DOAJ, REDIB, IRESIE, CLASE, DIALNET, SHERPA/ROMEO, QUALIS-CAPES, MIAR

Revista registrada en los directorios:

ULRICH'S, $\underline{\text { REDIE}}, \underline{\text { RINACE, OEI, MAESTROTECA, PREAL, CLACSO }}$ 


\section{Modelo de capital intelectual para la investigación en las universidades públicas de la Costa Caribe colombiana}

Intellectual capital model for research in public universities in the colombian Caribbean Coast

\section{Carlos Ramón Vidal Tovar ${ }^{1}$}

Resumen: El presente artículo propone un modelo de capital intelectual para la investigación en universidades públicas de la Costa Caribe colombiana. Para tal efecto, se realizó un estudio cuantitativo y de tipo analítico situacional con una población de siete (7) universidades conformada por 290 personas y una muestra de 79 sujetos informantes líderes de grupos de investigación categorizados y avalados por cada universidad objeto de estudio, a los cuales se les aplicó un cuestionario tipo Likert de 57 ítems. El análisis e interpretación de los datos permitió caracterizar en las universidades públicas de la Costa Caribe colombiana los activos intangibles presentes de forma favorable en los componentes de capital intelectual. Así como también, la presencia de los enfoques estratégico-corporativo y social evolutivo para la generación de capital intelectual desde la investigación. Lo anterior fue tomado como base para estructurar el modelo propuesto a partir de la presencia de treinta y cuatro variables o tipos de activos intangibles agrupados en once elementos o grupos de activos intangibles que forman parte del capital humano; el capital digital; el capital relacional formado por el capital social y el capital negocio; el capital estructural formado por el capital organizativo y el capital tecnológico.

Palabras clave: capital humano, universidad pública, investigación, capital intelectual.

Abstract: This article proposes a model of intellectual capital for research in public universities in the Colombian Caribbean coast. Thus, a quantitative research with a situational analytic type was performed with a population of seven (7) universities made up of 290 people and a sample of 79 informants subjects leading research groups categorized and endorsed by each university under study, to which they were applied a Likert questionnaire of 57 items. The analysis and interpretation of data characterizing allowed in public universities in the Colombian Caribbean coast intangible assets favorably present in the components of intellectual capital. As well as the presence of the evolving strategic and corporate and social approaches to generate intellectual capital from research. This was taken as a basis for the proposed structure from the presence of thirty-four variables or types of intangible assets grouped into eleven elements or groups of intangible assets that are part of the Human Capital model; Digital Capital; Relational Capital formed by the Social Capital and Business Capital; Structural Capital formed by the Organizational Capital and Technological Capital.

Key words: human capital, public university, research, intellectual capital.

\footnotetext{
${ }_{1}$ Docente del Programa de Ingeniería Agroindustrial. Universidad

Popular del Cesar, en Valledupar, Cesar, Colombia.
}

Dirección electrónica: carlosvidal@unicesar.edu.co

vidal.tovar@gmail.com

Artículo recibido: 23 de marzo, 2016

Enviado a corrección: 19 de octubre, 2016

Aprobado: 5 de diciembre, 2016 


\section{Introducción}

El siglo 21 ha traído consigo la aceleración de muchos procesos en el desarrollo de las organizaciones por efecto de la disponibilidad de la información y la apertura de fronteras a la comercialización de bienes materiales u otros insumos necesarios en un sin número de mercados. Acorde con Farfán y Garzón (2006), actualmente se vive una revolución que, lejos de ser una moda pasajera, es el producto de fuerzas incontrolables sin reversa: la globalización, la informatización, la falta de intermediación económica y la intangibilización, factores que han producido en las organizaciones un repensar en la valoración de sus activos reflejados en sus resultados contables y tener en cuenta el capital intelectual generado en sus procesos como medida de su competitividad y éxito en el entorno comercial y social donde se desenvuelve.

En este sentido, las universidades no pueden ser ajenas a la necesidad de crecer acorde con el desarrollo social, económico, cultural y ambiental donde se desenvuelven; la justificación pertinente de sus programas desde lo académico las fortalece en lo social, pero la investigación es la función que le permite a la universidad integrarse a las dimensiones de su entorno y a la sociedad como factor decisivo del desarrollo económico a la medida que produzca nuevo conocimiento científico y tecnológico fundamentado en las necesidades sociales, institucionales, organizacionales y productivas, generando capital intelectual entre sus miembros y haciéndolas más competitivas frente a las exigencias propuestas en el mundo globalizado actual.

De esta forma, la universidad como fuente del saber y cultura, motor de la nueva sociedad del conocimiento e impulsada a desarrollar ciencia y tecnología por medio de la investigación, produce activos intelectuales o de conocimiento, cuya naturaleza es intangible como son los conocimientos que poseen las personas, sus talento, ideas, patentes, invenciones, sistemas, aplicaciones y demás tipo de trabajo creativo que se puede identificar, reconocer, definir, valorar, medir, de uso específico y concreto en la organización y propio del sujeto de conocimiento estudiado; es lo que se conoce como capital intelectual (Seguí, 2007).

Desde el escenario anterior, los modelos de capital intelectual son una herramienta fundamental para la gestión de las ventajas competitivas que pueden obtener las universidades al tomar como base la valoración del capital intelectual generado por la investigación, teniendo en cuenta los activos actualmente críticos en la cadena de valor de bienes y servicios: los intangibles (Seguí, 2007); por lo que es necesario para las 
universidades tener un modelo de capital intelectual definido acorde con sus necesidades con el fin de valorar los intangibles producidos en la dinámica de la investigación.

Como ejemplo a nivel mundial cabe destacar el caso de Austria, donde se retoma la experiencia en centros de investigación, para sustentar la reforma universitaria del 2002 y emitir la ley sobre la reorganización de todas las universidades públicas austriacas denominada "UG 2002" la ley señala que: la declaración del capital intelectual deberá servir como un instrumento de gestión para las universidades, así como un instrumento de comunicación entre universidades y el propio ministerio (Leitner, 2004). De igual forma, Bueno (2003a), catedrático de economía de la empresa y director del centro de investigación sobre la sociedad del conocimiento (CIC) de la Universidad Autónoma de Madrid, España, propone en el 2003 el modelo Intellectus para la valoración de los activos intangibles, medición y gestión del capital intelectual en universidades y organismos públicos de investigación (OPI's).

A nivel latinoamericano, Bustos (2008), en México D.F., realiza un análisis de los modelos de capital intelectual aplicados en el proceso de producción del conocimiento y la gestión del capital intelectual en las universidades y centros de investigación; cinco en el ámbito europeo y uno en el latinoamericano. Tres como propuestas teóricas y tres que incluyen experiencias empíricas; destacando a Fazlagic (2005), Hazelkorn (2005), Leal de Suárez (2003), Leitner (2004) y Sánchez, Castrillo y Elena (2006).

Así mismo, es significativo lo realizado en las instituciones mexicanas como el Instituto Tecnológico y de Estudios Superiores de Monterrey (ITESM), la Universidad Nacional Autónoma de México (UNAM) y el Instituto Politécnico Nacional (IPN), analizadas por autores como Carrillo (2000), Funes (2007), Molina (2001) y Rivas (2004) y en Venezuela, Leal de Suárez (2003), propone un sistema de treinta y cuatro indicadores para evaluar la gestión del capital intelectual en las organizaciones universitarias, desde los procesos de planificación, organización, dirección y control (Bustos, 2008).

En el mismo sentido, en Colombia, se pueden destacar los siguientes estudios:

1. "Estado actual del capital intelectual y la gestión del conocimiento en las universidades del Risaralda", el cual se orienta al reconocimiento de los intangibles desarrollados en el ámbito local de las universidades, fue realizado por un grupo de investigadores encabezados por Ramón Albeiro Hernández Valencia (Hernández, Cardona, Cardona y Lasso, 2007).

2. "Análisis del valor agregado del conocimiento. Caso aplicado en una institución de educación superior". Se muestra un caso de estudio aplicado a una empresa del sector 
educativo de Colombia, de cómo aplicar dicha metodología para la valoración del capital intelectual; la investigación fue realizada por un grupo de profesionales encabezado por Paula Andrea Molina Parra (Molina, Arango y Botero, 2010).

3. "La investigación en UNIANDES: construcción de una política". Se presenta un ejercicio de estimación del esfuerzo financiero total que hace la Universidad de los Andes para desarrollar tareas de investigación. Se reconoce a los grupos de investigación como organizaciones que no tienen personería jurídica, el estudio fue liderado por Villaveces (2010).

4. "Gestión del capital intelectual de las instituciones educación superior. Caso Universidad Nacional de Colombia - sede Manizales". Se presenta un análisis realizado cuyo propósito fue conocer el estado de la gestión del capital intelectual de las instituciones de Educación Superior. La investigación fue realizada por Mosquera (2011).

Lo anterior demuestra el interés de muchas universidades colombianas y en especial las de la Costa Caribe, en conocer y valorar cómo se dinamiza el capital intelectual producido, lo cual es de gran importancia para una ciudad, para una Región y para un país con el fin de estar en constante mejoramiento de la calidad con que se construye el conocimiento en las aulas de las universidades para generar ventajas competitivas como, mayor cobertura, accesos a muchos privilegios como convenios interinstitucionales, financiación de proyectos y reconocimiento en su entorno. Sin embargo no muestran un modelo determinado de capital intelectual implementado que oriente los procesos del desarrollo, producción y valoración de los intangibles obtenidos desde la investigación.

Bajo el escenario planteado, las universidades localizadas en la Costa Caribe de Colombia necesitan de un modelo de capital intelectual que defina su enfoque y componentes intangibles necesarios para su orientación acorde a los procesos de investigación para desarrollar un conjunto de productos o activos intangibles y competencias en sus investigadores; su organización con el entorno socio económico-productivo; los medios, herramientas y estructura física necesaria para aumentar la valoración de sus indicadores genéricos y específicos.

En consecuencia, es de suma importancia para las universidades públicas de la Región Caribe de Colombia saber cuál es el modelo por seguir de capital intelectual para identificar, medir y evaluar estos activos intangibles con el fin de orientar la planificación, dirección y gestión del conocimiento implicado, al igual que diseñar y desplegar políticas científicas y planes de investigación con mayores dosis de eficiencia, buscando un equilibrio y equidad sistémica que facilite el buen logro de posibles estrategias relacionadas con 
desarrollo y excelencia de la capacidad investigadora exigida por el ministerio de educación nacional.

De esta forma, a continuación, se presenta la estructura teórica sobre la cual se fundamenta el presente estudio. Así mismo, se analiza la opinión de diferentes autores desde diversos tópicos orientados al capital intelectual.

\section{Breve referente teórico}

\subsection{Capital intelectual}

Para Bueno (2003b), el capital intelectual lo define como el conjunto de activos intangibles que generan y generarán valor para la organización en el futuro. Los conocimientos de las personas, sus capacidades, talento y destrezas, el reconocimiento de la sociedad, la calidad de las relaciones que se mantienen entre los miembros y equipos pertenecientes a otras organizaciones, etc., son algunos de los activos intangibles que muestran gran parte de la valoración realizada por la sociedad y la comunidad científica a una universidad o centro de investigación. Es decir, todos los activos intelectuales o de naturaleza intangible (Bueno, 2003b, p. 13).

Igualmente, Farfán y Garzón (2006) define el capital intelectual como el conjunto de recursos intangibles de la organización que tienen la capacidad de generar valor ya sea en el presente, y en el futuro. Se trata de todos aquellos recursos considerados en un sentido amplio y no solo estrictamente en el sentido contable. En otras palabras, incluye los activos intangibles que la norma contable permite reconocer, así como también las capacidades o habilidades de los empleados y la organización.

Igualmente, Cobo (2006) define el capital intelectual como la combinación de activos intangibles o inmateriales, algunos inagotables como el conocimiento de las personas, su forma de aprender, la capacidad de adaptación al entorno, relaciones con los clientes, nombres de los productos, proveedores, las patentes, las marcas, administraciones, reputación de la empresa, los procesos internos y la capacidad de investigación, desarrollo e innovación de una organización que no se ven normalmente en los estados contables tradicionales, pero generan valor en el presente y futuro.

En este sentido, Seguí (2007) realiza un análisis de diferentes autores para afirmar que los conceptos de gestión del conocimiento y gestión del capital intelectual se pueden tratar de forma similar y que la diferencia radica en el enfoque metodológico de cada autor. Específicamente, el capital intelectual se refiere a la perspectiva global y estratégica de los 
activos intelectuales de una empresa, es la suma del conocimiento de sus miembros y de la interpretación práctica. Es un fenómeno complejo, que resulta de una práctica colectiva, que crea valor a través de sus relaciones e interrelaciones entre recursos, competencias y capacidades organizacionales (Da Silva, Figueroa y Fernández-Jardón, 2009).

De igual forma, Da Silva Santos y otros (2009) afirman que el capital intelectual en sentido amplio, es utilizado de forma sinónima con términos como: activo intelectual, intangible u oculto, goodwill, o propiedad intelectual; muy parecido con lo definido por Bueno (2003b, p. 13) refiriéndose a todos aquellos activos intelectuales o de conocimiento de naturaleza intangible (como son los conocimientos poseídos por las personas, talento, ideas, invenciones, patentes, sistemas, aplicaciones y todo tipo de trabajo creativo) que se puedan identificar, definir, medir y valorar para una organización.

\subsection{Componentes del capital intelectual}

Bueno (2003b) agrupa los activos intangibles según su naturaleza en tres componentes: capital humano, capital estructural y capital relacional. Subdivide el capital estructural, en capital organizativo y capital tecnológico. Al capital relacional lo divide en capital negocio y capital social. Las razones para descomponer el capital estructural y el capital relacional se fundamentan en la propia evolución de los conceptos involucrados y por la observación de la emergencia de determinadas prácticas, consideradas como relevantes y pioneras en materia de gestión del conocimiento (Cobo, 2006).

En este sentido, en los últimos, años la dinámica del conocimiento ha generado numerosos modelos para la gestión del capital intelectual; lo común de ellos es la clasificación que realizan de los componentes del capital intelectual según su naturaleza, lo que representa la combinación de los recursos humanos, organizativos y relacionales de una universidad. De este modo, se puede descomponer el capital intelectual en: capital humano, capital estructural y capital relacional. El capital intelectual es más que la suma de los componentes individuales, ya que la conectividad de los 3 elementos citados también puede ser fuente de valor para la organización (Seguí, 2007). Como consecuencia de estas relaciones existentes entre los distintos componentes del capital intelectual, cualquier estrategia que tenga como finalidad su desarrollo en la organización, debe tener en cuenta las relaciones existentes entre los distintos componentes de capital intelectual, para efectuar las acciones que hagan que este capital sea mayor y más conveniente para la organización (Cobo, 2006). 
En ese orden, para una organización universitaria, Bustos (2008) afirma que el capital intelectual lo constituyen todos los trámites y activos invisibles de la compañía; se puede dividir en capital estructural y capital humano. Estos dos parámetros representan lo que se denomina como los activos invisibles y el conocimiento encarnado en los empleados de la universidad. La separación entre el capital intelectual "pensante" y el "no pensante" se puede considerar como el criterio de diferenciación a este nivel. La razón es que las personas y, por tanto, el capital humano necesitan unos métodos de gestión completamente distintos al del capital estructural.

Continuando con Bustos (2008), la mayoría de los modelos de capital intelectual asumen una distinción tripartita entre: la estructura externa, la estructura interna y los empleados. Así, el capital intelectual se compone y está generado de una parte pensante (el capital humano) y de una parte no pensante (el capital estructural). El capital estructural tiene un componente interno y otro externo (capital organizativo y de relaciones respectivamente), mientras que el capital humano proviene del conocimiento, de la actitud y de la agilidad intelectual de los empleados.

Sumado a lo anterior, la era digital o era de la economía digital, ha tenido influencia indiscutible debido a la utilización de la internet como medio de integración del capital humano con el capital relacional y el capital estructural, con el fin de crear valor por medio de la promoción de una nueva plataforma de competencias, extrayendo el poder de las redes de negocios o business webs originando la inclusión de un componente más al capital intelectual denominado "capital digital" (Tapscott, Ticoll y Lowy, 2000).

\subsection{Aspectos normativos de la investigación en la universidad colombiana.}

El marco legal del sistema de educación superior de Colombia es soportado principalmente en la ley 30 de 1992, ley 115 de 1994, ley 749 de 2002, la ley 1188 del 2008 y el decreto 1295 de 2010; dicho sistema está constituido por entes reguladores, evaluadores, asesores y prestadores de servicio público; donde los reguladores son el Congreso de la República, el Ministerio de Educación Nacional y el Departamento Nacional de Planeación. Estas entidades cumplen funciones de evaluación, además de otros organismos como el Consejo Nacional de Educación Superior -CESU, Consejo Nacional de Acreditación -CNA, Consejo Nacional de Aseguramiento de la Calidad de la Educación Superior -CONACES. Los evaluadores y asesores son el Sistema Nacional de Información de la Educación Superior -SNIES; Sistema para la Prevención y Análisis de la Deserción en las Instituciones 
de Educación Superior -SPADIES; el Observatorio Laboral para la Educación y los pares académicos.

El sistema mencionado es el encargado de vigilar y hacer cumplir a las universidades (prestadores del servicio público, entidades de educación superior pública y privada) con sus funciones sustantivas de docencia, proyección social y en especial la investigación; tomando como base tres factores contemplados por el Consejo Nacional de Acreditación -CNA (2009), los cuales se complementan y se fortalecen mutuamente como son:

1. El espectacular avance logrado en algunos campos del conocimiento, lo cual ha acortado dramáticamente la distancia entre el laboratorio y la línea de producción.

2. Se está pasando por una transición de la era de sistemas de producción basados eminentemente en recursos físicos y energéticos, a sistemas de producción basados en el conocimiento, buscando más eficiencia y productividad desde su utilización y capacidad de generación.

3. Los desafíos que hoy se confrontan en términos de sostenibilidad económica (generación de empleo), sostenibilidad social (asegurar un desarrollo con inclusión social) y sostenibilidad ambiental.

Por otra parte, se encuentra la ley 1286 de 2009, la cual transforma a Colciencias en Departamento Administrativo y fortalece el Sistema Nacional de Ciencia, Tecnología e Innovación en Colombia como organismo principal de la administración pública, rector del sector y del Sistema Nacional de Ciencia, Tecnología e Innovación -SNCTI-, encargado de formular, orientar, dirigir, coordinar, ejecutar e implementar la política del estado en la materia en concordancia con los planes y programas de desarrollo. Entre las funciones de Colciencias se pueden mencionar: realizar el seguimiento y evaluación de la política nacional de ciencia, tecnología e innovación; promover y evaluar la alianza estratégica universidadempresa; fomentar y consolidar, con visión de largo plazo en las instituciones de educación superior los centros y grupos de investigación; hacer prospectiva en ciencia, tecnología e innovación; adoptar decisiones y emprender acciones en materia de ciencia, tecnología e innovación.

Además, Colciencias es el encargado de administrar el software encargado de dar a conocer la producción científica a nivel nacional por la valoración de indicadores de ciencia y tecnología producidos por diferentes instituciones en su mayoría por instituciones de educación superior utilizando los siguientes aplicativos:

CvLAC: Currículum Vitae Latinoamericano y del Caribe, es un software basado en un proyecto estratégico de cooperación técnica de la coordinación de investigaciones de la 
Organización Panamericana de la Salud (OPS), la cual por iniciativa de Colciencias en colaboración con el Grupo Académico CT\&S-UN de La Universidad Nacional de Colombia y la Corporación de Ciencia, Tecnología y Sociedad CT\&S desarrolló el aplicativo como un espacio común de integración e intercambio de información de los currículos de todas aquellas personas que forman parte de los sistemas de ciencia y tecnología de los países que participan en dicho espacio (Colciencias, 2014).

GrupLAC: Grupo Latinoamérica y del Caribe, es un software originalmente desarrollado por el grupo Stela de la Universidad Federal de Santa Catarina, cuyo objetivo es mantener un directorio de los grupos de investigación, instituciones e investigadores que participan activamente en el desarrollo de nuevas estrategias en el ámbito de la ciencia, la tecnología y la innovación, utilizado en Colombia desde el 2002 para almacenar la información proveniente de los grupos de investigación del país y es registrada por los líderes en cada uno de ellos (Colciencias, 2013).

InstituLAC: Es un software de Colciencias, desarrollado para ver y crear un amplio directorio de instituciones, a las cuales están vinculados los diferentes grupos de investigación y los diferentes investigadores de instituciones participantes en la red del sistema nacional de ciencia, tecnología e innovación (Colciencias, 2012).

De esa forma, en Colombia los productos de investigación se hacen visibles en bases de datos con indicadores estadísticos confiables sobre investigaciones desarrolladas por instituciones, grupos de investigación, centros de investigación e investigadores; en ellas, se puede obtener información en tiempo real. Estas bases de datos dan a conocer los indicadores de la producción científica de la actividad investigativa en las universidades, para lo cual se hace una convocatoria donde se actualizan los currículos de investigadores por medio del CVLAC y se categorizan los grupos de investigación acorde con su trascendencia en la investigación y el nivel de profundidad de las investigaciones realizadas.

Por ello, para las universidades es significativo e importante la visualización y categorización de sus grupos de investigación en el Sistema Nacional de Ciencia, Tecnología e Innovación -SNCTI- tomando como base los productos de investigación reportados, la calidad de estos y la madurez o trascendencia del grupo. Lo cual da a las universidades un estatus en el escalafón a nivel nacional y así acceder a mejores fuentes de financiación para la investigación por medio de sus grupos en diferentes convocatorias.

De esta forma, el sistema descrito anteriormente muestra cómo es valorada la universidad por medio de la productividad científica tangible reportada por sus grupos de investigación mas no permite observar la forma cómo lo hacen, el enfoque aplicado en los 
procesos de gestión de la investigación en la universidad o cuál es el modelo aplicado para dinamizar los procesos que conllevan a los grupos de investigación a obtener productos de investigación que cumplan con las expectativas del Sistema Nacional de Ciencia, Tecnología e Innovación; solo cuantifica los productos logrados por los grupos sin tener en cuenta las estrategias desarrolladas por la universidad para que los grupos por medio de sus investigadores produzcan capital intelectual de forma ordenada y bajo unas directrices a favor de la institución de educación superior; lo cual genera disparidad al momento de comparar las universidades por sus indicadores de producción científica.

De igual forma, se intuye que las universidades mencionadas anteriormente poseen o deben poseer una riqueza de conocimiento o un modelo de capital intelectual que permite justificar el mayor o menor potencial investigador disponible y determine el camino por seguir para continuar produciendo científicamente mediante la puesta en acción a través de los correspondientes procesos cognitivos, los activos intelectuales o intangibles existentes; que de continuar invisibles nunca serán conocidas las fortalezas de estas instituciones para generar capital intelectual desde la investigación. Por tal motivo, esta investigación se fundamentó en los siguientes interrogantes:

¿Cómo podría ser el modelo de capital intelectual para la investigación en las universidades públicas de la Costa Caribe colombiana?

¿Cuáles son las características de los componentes del capital intelectual utilizado para la investigación en las universidades públicas de la Costa Caribe colombiana?

¿Qué tipo de enfoque tiene el modelo aplicado para la investigación en las universidades públicas de la Costa Caribe de Colombia?

\section{Metodología}

Para dar respuesta a los anteriores interrogantes, se desarrolló un estudio cuantitativo y de tipo analítico en la forma como las universidades públicas de la Costa Caribe de Colombia desarrollan sus procesos de investigación, para lo cual fue necesario la caracterización de los componentes del capital intelectual utilizados; la identificación del enfoque del modelo aplicado y la descripción de los componentes presentes en el capital intelectual generado por los investigadores de siete universidades públicas con el fin de proponer un modelo de capital intelectual para la investigación en estas universidades. 


\subsection{Participantes, instrumento y recolección de datos.}

La población estuvo conformada por 290 líderes de grupos de investigación de siete (7) universidades públicas localizadas en la Región Caribe o Costa Norte de Colombia, como son, la Universidad de la Guajira, Universidad Popular del Cesar, Universidad de Sucre, Universidad de Córdoba, Universidad del Atlántico, Universidad del Magdalena y la Universidad de Cartagena; donde fueron seleccionados por medio de un muestreo probabilístico estratificado a 79 líderes de grupos de investigación como informantes de las universidades mencionadas anteriormente.

De esta forma, para darle cumplimiento al objetivo propuesto en esta investigación se aplicó a los líderes de grupo de investigación seleccionados una encuesta como técnica de recolección de datos, por medio de un cuestionario conformado por 57 ítems que valoraron la variable capital intelectual a través de sus dimensiones componentes y enfoque del modelo; las preguntas estuvieron centradas en las subdimensiones capital humano, capital estructural, capital relacional y capital digital desde los indicadores: formación de investigadores, interacción entre investigadores, recursos financieros disponibles, dotación tecnológica, relaciones con empresa para desarrollar investigación, relaciones con otras universidades para desarrollar investigación, repositorios virtuales y redes de conocimiento. A los líderes de grupos de investigación antes de realizar la encuesta se les hizo saber que la información suministrada sería procesada en conjunto, por lo que en ningún caso se publicarían en forma individual, lo cual fue aceptado sin objeciones.

El cuestionario se estructuró con una escala de Likert; cada ítem o pregunta ofreció cinco alternativas de respuesta tomando su ponderación en 5 categorías: totalmente de acuerdo, parcialmente de acuerdo, neutral, parcialmente en desacuerdo y totalmente en desacuerdo. El cuestionario fue validado por siete expertos del doctorado en Ciencias, mención Gerencia de la Universidad Rafael Belloso Chacín en Maracaibo, Venezuela. La confiabilidad fue calculada a partir de una prueba piloto a veinte sujetos y se calculó el coeficiente de alfa Cronbach, obteniendo un resultado de 0,952, lo cual es de una confiabilidad elevada. Los datos obtenidos fueron tratados estadísticamente a partir del cálculo de la media, la frecuencia absoluta y la frecuencia relativa.

En ese sentido, los resultados fueron interpretados utilizando el siguiente baremo: todas las respuestas elegidas como totalmente de acuerdo y parcialmente de acuerdo fueron consideras como presencia favorable con una frecuencia relativa entre $50 \%$ y $100 \%$; las respuestas parcialmente en desacuerdo y totalmente en desacuerdo fueron consideras como 
presencia desfavorable con una frecuencia relativa entre $0 \%$ y $49 \%$. Las respuestas neutrales se analizaron tomando como base el valor de $15 \%$ correspondiente a la media obtenida de todas las respuestas neutrales dadas por los informantes. Indicadores con valores superiores a este fueron considerados estadísticamente significativos y analizados de forma particular para cada dimensión.

\section{Resultados}

A continuación se presentan los resultados sistematizados producto de la información suministrada por los líderes de grupos de investigación en 2 graficas de frecuencia y el análisis de cada una de ellas; la primera para la dimensión componentes de capital intelectual y las subdimensiones capital humano, capital estructural, capital relacional y capital digital. La segunda corresponde a la dimensión enfoques del modelo aplicado para la investigación en las universidades públicas de la Costa Caribe de Colombia; con las subdimensiones enfoque financiero administrativo, enfoque estratégico corporativo y enfoque social evolutivo.

Lo anterior permite analizar de forma lógica la variable de estudio, contrastarla con las teorías tomadas como fundamentos para la formulación de los objetivos propuestos y generar el modelo de capital intelectual para la investigación en las universidades públicas de la Costa Caribe colombiana, su fundamentación teórica, objetivos, estructura e indicadores para su reconocimiento y valoración.

\subsection{Componentes de capital intelectual en universidades de la Costa Caribe colombiana}

El grafico uno muestra que el capital humano presenta una presencia favorable del 59 \% teniendo en cuenta la formación de investigadores y la interacción entre investigadores. Dado que el capital humano se considera como el valor de los conocimientos y el talento que tienen los investigadores de la universidad, vinculados a ella mediante contratos laborales o nombramientos de funcionarios, no solamente de carácter formal y explícitos, sino de carácter psicosocial (Cobo, 2006). 


\section{Grafico 1}

Frecuencia relativa de la favorabilidad de los componentes de capital intelectual presentes en universidades de la Costa Caribe colombiana

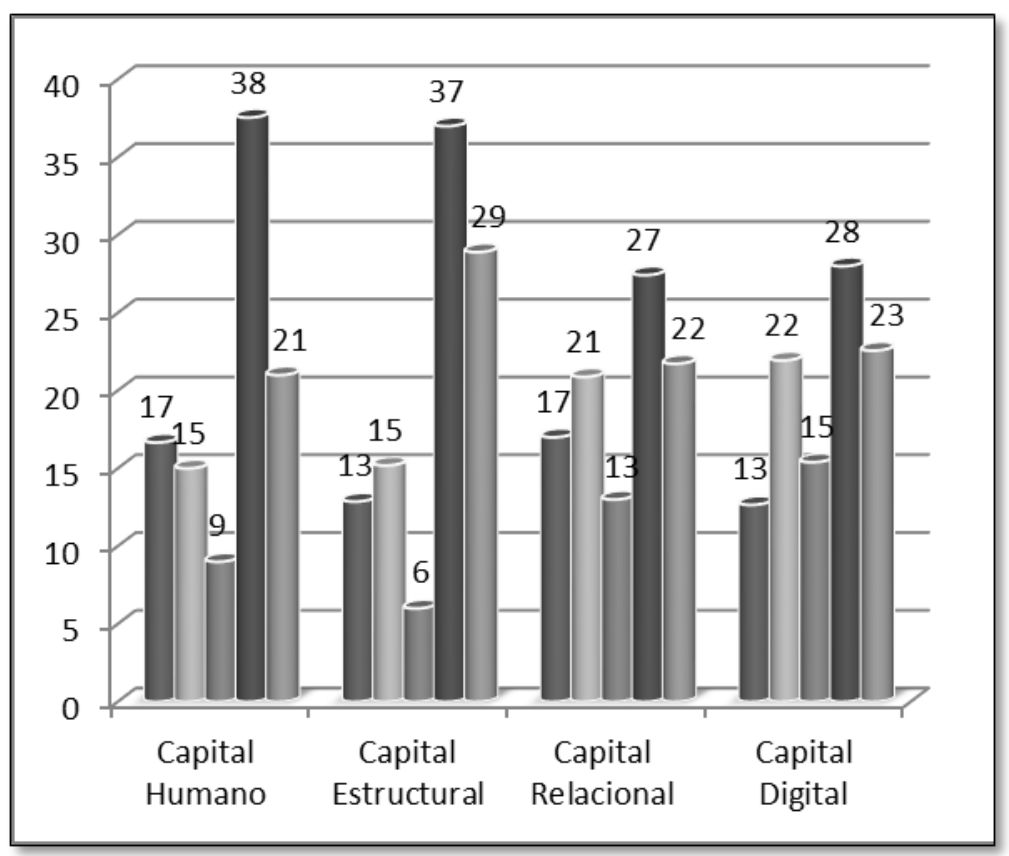

Fuente: Elaboración propia (2014). Evaluación de los resultados de la encuesta realizada a investigadores de las universidades públicas localizadas en la Costa Caribe de Colombia

Así mismo, el capital estructural presenta una presencia favorable de 66 \% basado en los recursos financieros disponibles y la dotación tecnológica destinada a la investigación, además proviene del valor organizativo y de relación, refleja los focos externos e internos de la universidad, más el valor de renovación y de desarrollo, es decir, el potencial para el futuro según Bustos (2008).

Así mismo, el capital relacional presentó una presencia favorable del 49 \% teniendo en cuenta las relaciones con empresas públicas o privadas y con otras universidades para desarrollar investigación; se refiere al conjunto de relaciones económicas, políticas e institucionales que las universidades han desarrollado y mantienen con los diferentes agentes que configuran su entorno socioeconómico (Bueno, 2003a).

En ese orden, el capital digital tuvo una presencia favorable del $51 \%$ tomando como base los repositorios virtuales y las redes de conocimiento; surge por la necesidad de aprovechar la tecnología digital y la internet para dinamizar la interconexión del capital humano, el capital relacional o cliente y el capital estructural, originando una nueva fuente de sostener y generar ventajas competitivas por medio de la economía de la información, 
permite mejorar en gran medida la productividad y el rendimiento general en las organizaciones (Tapscott, 2000).

De igual forma, se puede observar en el grafico 1, la presencia desfavorable de los componentes mencionados anteriormente entre un $28 \%$ y $38 \%$, al igual que la actitud neutral asumida por los líderes de grupos de investigación frente al capital relacional con 13 $\%$ y el capital digital con $15 \%$. Lo anterior puede ser debido al desconocimiento y baja aplicación por algunos investigadores de los elementos que conforman los componentes mencionados como el trabajo en red para producir investigación, al igual que las estrategias digitales para aprovechar los productos de investigación generados.

\subsection{Enfoques del modelo aplicado para la investigación en las universidades públicas de la Costa Caribe de Colombia}

El grafico 2 permite apreciar como el enfoque financiero administrativo presenta una presencia desfavorable, mientras el enfoque estratégico corporativo y el social evolutivo poseen presencia favorable de su aplicación en las universidades de la Costa Caribe colombiana. Los resultados anteriores evidencian diferencias en las opiniones de los líderes de investigación, lo que permite inferir que las universidades de la Costa Caribe colombiana no tienen un enfoque definido para el desarrollo de la investigación y lo fundamentan tomando en cuenta el predominio del estratégico corporativo y en menor medida el social evolutivo.

Lo anterior se identifica con lo propuesto por Bueno, Salmador y Merino (2008) sobre capital intelectual al definirlo como la acumulación de conocimiento que crea valor o riqueza cognitiva poseída por una organización, compuesta por un conjunto de activos de naturaleza intangible o recursos y capacidades basados en conocimiento, que cuando se ponen en acción, según determinada estrategia, en combinación con el capital físico o tangible, es capaz de producir bienes y servicios, de generar ventajas competitivas o competencias esenciales para la organización en el mercado. 
Grafico 2

Frecuencia relativa de la favorabilidad de los enfoques del modelo aplicado para la investigación en las universidades públicas de la Costa Caribe de Colombia

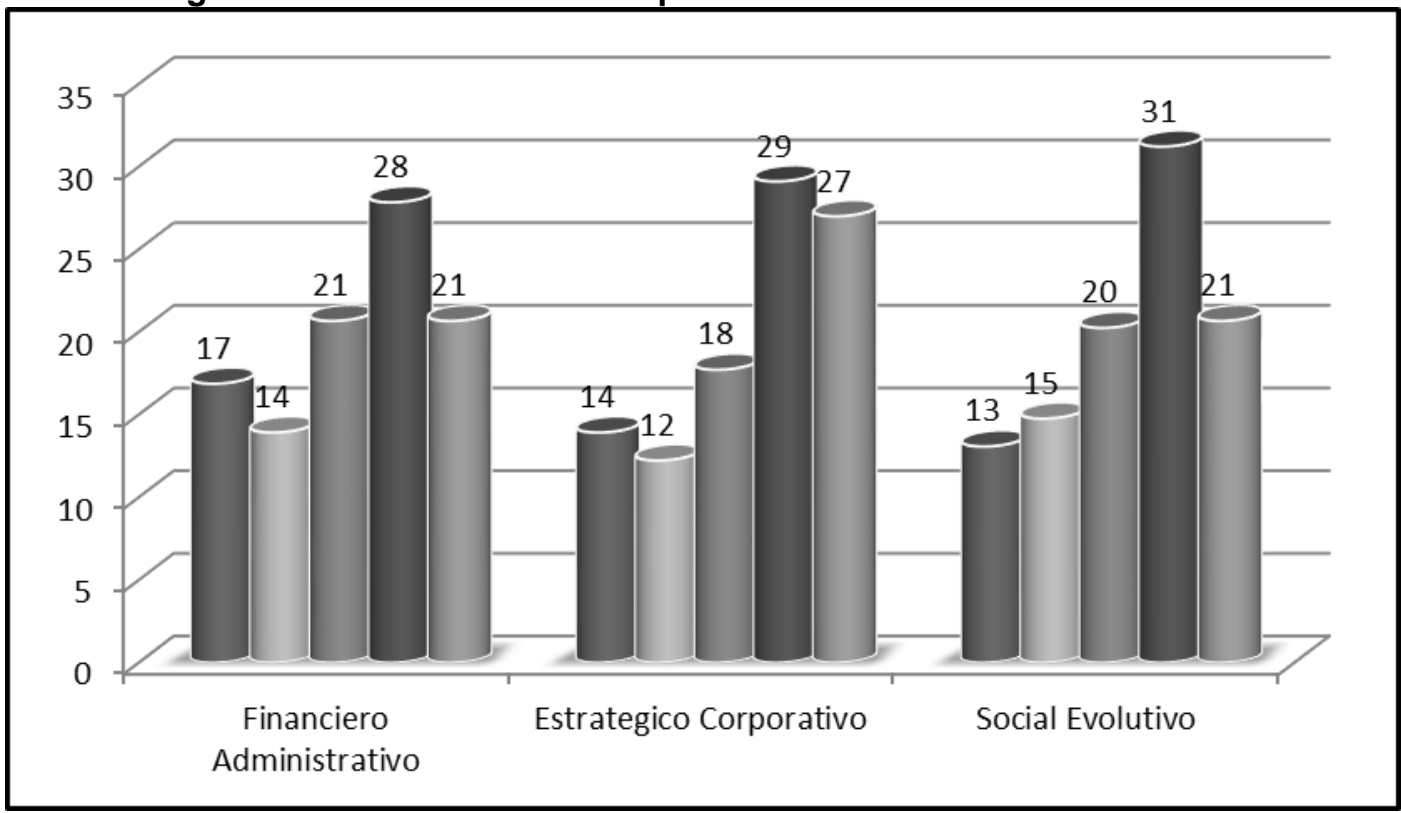

Fuente: Elaboración propia (2014). Evaluación de los resultados de la encuesta realizada a investigadores de las universidades públicas localizadas en la Costa Caribe de Colombia.

\subsection{Modelo de capital intelectual para la investigación en las universidades públicas de la Costa Caribe colombiana.}

Tomando como base los resultados arrojados por el análisis de los objetivos propuestos de este estudio, al igual que la revisión bibliográfica realizada desde el constructo teórico aportado por Tapscott (2000), Bueno (2003b), Cobo (2006), Seguí (2007) y Bustos (2008), entre otros, se procede a diseñar el modelo de capital intelectual para la investigación en las universidades públicas de la Costa Caribe colombiana destacando aspectos de suma importancia como los siguientes.

\subsubsection{Fundamentación teórica}

Para Cobo (2006), todo modelo debe mostrar, de forma propia, comprensible y estructurada, una serie de instrumentos de evaluación de intangibles de diferente naturaleza, con el fin de facilitar la información a terceros y a la dirección y gestión de la organización, ya que estos visibilizan el potencial futuro de la organización con el consiguiente aumento de la confianza de todos los agentes relacionados con ella. El modelo de capital intelectual debe expresar los objetivos que quiere conseguir, los usuarios a quien va dirigido, debe ser 
abierto, flexible y aplicable, debe medir no solo los resultados, sino también los procesos que los generan y recoger, como mínimo, información de los tres componentes principales como son capital humano, capital estructural y capital relacional (Cobo, 2006).

Igualmente, cabe aclarar que el modelo propuesto más adelante, se fundamenta en la estructura arbórea del modelo Intellectus (Bueno, 2003b), tomando sus componentes principales capital humano, capital estructural y capital relacional e incluyendo como aporte de este estudio el componente capital digital (Tapscott, 2000). Para cada componente se tienen en cuenta sus elementos o activos intangibles propios de cada universidad y los indicadores o forma como se pueden medir y evaluar en cada institución los elementos intangibles presentes.

En ese orden, el constructo teórico permite definir el capital intelectual, generado desde la investigación en las universidades, como el conjunto de activos intangibles producidos por la dinámica del conocimiento de los investigadores que conllevan a la agregación de valor, adición de ventajas competitivas y ventajas sostenibles para la universidad; sus grupos de investigación, equipos de trabajo y al investigador como tal, las cuales no se reflejan en los estados financieros o contables de la universidad, pero sí permiten su visibilidad y competitividad a largo plazo en la comunidad científica o el entorno donde se desarrollan.

Desde lo anterior, el modelo tiene como objetivo fundamental contribuir con las universidades de la Costa Caribe colombiana a la valoración y reconocimiento de sus activos intangibles que forman parte del capital intelectual generado desde la investigación por sus participantes. A continuación se presentan los componentes o capitales integrantes del modelo de capital intelectual para la investigación en las universidades públicas de la Costa Caribe colombiana; la conceptualización de cada componente, sus indicadores, características y estructura de cada uno de ellos.

\subsubsection{Componentes del modelo}

\subsubsection{Capital humano}

El capital humano es el cumulo de conocimientos construidos por un investigador a través de diferentes procesos de enseñanza aprendizaje o procesos de formación, alimentados por sus experiencias, competencias, capacidades y talentos con el fin de adicionar valor intangible a un grupo de investigación, equipo de trabajo, a la universidad o a el mismo. Esto es propio de cada investigador y las universidades están en riesgo de perderlo al concluir el vínculo contractual con el investigador por no sistematizar, socializar y 
poner en práctica su conocimiento. El capital humano de las universidades públicas de la Costa Caribe colombiana está compuesto por los siguientes activos intangibles:

Formación de investigadores. Corresponde a los estudios de posgrados ofertados por la universidad donde se promueve una formación indispensable para la investigación y al apoyo recibido por los investigadores para incrementar su nivel de formación investigativa. Se evalúa por los indicadores oferta de estudios de maestría y doctorado en investigación, programas de actualización en investigación desarrollados y apoyo a docentes para realizar estudios de postgrado en investigación dentro o fuera del país.

Interacción entre investigadores. Proceso de aprendizaje logrado por estrategias de la universidad o por iniciativa de los investigadores para intercambiar conocimientos entre investigadores e incrementar sus saberes de temas determinados. Se evalúa tomando como base los eventos o espacios de interacción desarrollados en la universidad, participación conjunta de varios grupos en la ejecución de proyectos de investigación, ya sea con grupos de la misma universidad, grupos nacionales o extranjeros y desarrollo de eventos de socialización de productos de investigación de la misma forma. La tabla 1 muestra los indicadores para medir el capital humano.

Tabla 1

Indicadores para medir el capital humano Indicador

Número de programas de posgrados ofrecidos en investigación / Número de programas de postgrados ofrecidos por la universidad

Número de programas de actualización en investigación desarrollados / Número de programas de actualización desarrollados para docentes

Número de docentes apoyados para realizar estudios de posgrado en investigación/ Número de docentes apoyados para realizar estudios de postgrado

Número de proyectos de investigación formulados por interacción entre investigadores / Número de proyectos de investigación formulados

Número de proyectos ejecutados entre varios grupos de investigación / Número de proyectos ejecutados

Número de eventos realizados para dar a conocer los productos de investigación

Fuente: Elaboración propia (2014). Producto del análisis de la información desde los resultados de la encuesta realizada a investigadores en las universidades públicas de la Costa Caribe de

Colombia.

\subsubsection{Capital estructural}

Se refiere a todos los procesos organizativos y a la infraestructura utilizada por investigadores para desarrollar sus investigaciones y generar capital intelectual desde la investigación en las universidades. En él se incluyen los procesos y procedimientos formales y sistematizados propios de la universidad así como también los activos tangibles logrados desde la investigación. Está formado por el capital organizativo y el capital tecnológico. 
Capital organizativo. Corresponde a los procesos organizacionales formales y estructuras administrativas y financieras que coadyuvan en la universidad para desarrollar la investigación. Lo conforman los siguientes activos intangibles:

Procesos organizativos. Todas aquellas actividades 0 acciones organizativas estructuradas y formales que permiten crear y desarrollar capital intelectual desde la investigación en las universidades. Es evaluado teniendo en cuenta los procesos de financiación a proyectos de investigación, procesos de seguimiento para el cumplimiento de proyectos de investigación y procesos para publicar la productividad científica de investigadores.

Recursos financieros disponibles. Son todos aquellos fondos monetarios o recursos monetarios destinados al desarrollo de la investigación en las universidades; es decir, la inversión económica utilizada para formular y ejecutar proyectos de investigación. Los recursos pueden provenir de las mismas universidades, de instituciones públicas o privadas, nacionales o internacionales. Es valorado por tener un presupuesto suficiente destinado a la investigación, recursos financieros procedentes de Colciencias para desarrollar investigación y por la disposición de recurso económico externo diferente al de Colciencias para desarrollar investigación.

Capital tecnológico. Conjunto de recursos técnicos y tecnológicos necesarios para desarrollar investigación, de participación directa en la generación de capital intelectual, utilizado por docentes y estudiantes vinculados a la práctica investigativa. Conformado por los siguientes activos intangibles:

Infraestructuras básicas. Se refiere a los recursos materiales propiedad de las universidades y disponibles para los investigadores con el fin de optimizar su rendimiento intelectual y con ello, generar capital intelectual desde la investigación, tales como, capacidad instalada en cuanto a edificios, laboratorios y ampliaciones estructurales destinadas a la investigación. Se evalúa por los activos tangibles logrados desde la investigación, laboratorios de uso exclusivo para la investigación y cubículos para el uso exclusivo de investigadores

Dotación tecnológica. Infraestructura conformada por instrumentos, equipos, medios técnicos y tecnologías involucrados en la formulación, ejecución y socialización de proyectos de investigación que coadyuvan a la generación de capital intelectual en las universidades. Se evalúa por la promoción de convocatorias actualizadas sobre investigación en el sistema de información de la universidad, la utilización del correo electrónico institucional como medio para comunicar actividades de investigación y la disponibilidad del internet en los 
sitios de trabajo de investigadores. La tabla 2 muestra los indicadores para medir el capital estructural.

Tabla 2

Indicadores para medir el capital estructural Indicador

Número de procesos formales establecidos para la financiación a proyectos de investigación

Número de procesos formales de seguimiento para el cumplimiento de proyectos de investigación

Número de procesos definidos para publicar la productividad científica de investigadores

Total en pesos desembolsado para proyectos de investigación ejecutado / total en pesos de proyectos aprobados por convocatoria

Total en pesos de recurso procedente de Colciencias para apoyar proyectos de investigación / total en pesos de recurso destinado para apoyar proyectos de investigación en la universidad

Total recurso en pesos procedente de fuentes externas diferentes a Colciencias para apoyar proyectos de investigación / total en pesos de recurso destinado para apoyar proyectos de investigación en la universidad

Número de activos tangibles obtenidos desde la investigación

Número de laboratorios dedicados solo a la labor de investigación

Número de sitios o cubículos disponibles para el desarrollo del trabajo de investigadores / Número de investigadores

Número de consultas en el sistema de información de la universidad sobre convocatorias para presentar proyectos de investigación

Número de usuarios investigadores de correos electrónicos institucionales activos

Número de puntos de conexión disponibles para investigadores en sus sitios de trabajo

Fuente: Elaboración propia (2014). Producto del análisis de la información desde los resultados de la encuesta realizada a investigadores en las universidades públicas de la Costa Caribe de

Colombia.

\subsubsection{Capital relacional}

Se refiere a los activos intangibles producidos por las relaciones establecidas entre la universidad y cualquier agente externo a ella, teniendo en cuenta el punto de encuentro desde el objeto social de ambas para establecer el vínculo entre las dos o más organizaciones a favor del desarrollo de la investigación. Está conformado por el capital social y el capital negocio.

Capital social. Se refiere a la manera como la universidad se hace visible e impacta desde el cumplimiento de sus funciones sustantivas: docencia, investigación y extensión en la sociedad. Desde la investigación, al conjunto de valores intangibles agregados a la institución a partir de las relaciones establecidas para desarrollar investigación y por los impactos de sus resultados en su entorno productivo y social. Está formado por los siguientes activos intangibles:

Integración con la comunidad científica. Interacción de los programas de formación desde cada una de sus áreas disciplinares, sus proyectos y productos de investigación con grupos de profesionales externos, reconocidos, visibles ante la comunidad científica y la 
sociedad, con el fin de socializar avances en áreas de acuerdo común y direccionar posibles rutas de profundización. Es evaluado por el apoyo económico otorgado a investigadores para participar en eventos de socialización de productos de investigación, eventos organizados por la universidad para dar a conocer productos de investigación y a la participación en asociaciones de tipo científico por sus productos de investigación.

Desarrollo de proyectos de impacto social. se refiere a la realización o participación de investigadores en trabajos de innovación realizados en torno a convocatorias internas o externas a la universidad, de forma independiente o en colaboración con otros agentes con el fin de ejecutar proyectos que contribuyen al mejoramiento del sector productivo o las condiciones de vida de la población. Lo que le otorga a la universidad un valor agregado intangible en el medio donde se desenvuelve. Es evaluado desde la ejecución de proyectos de investigación que contribuyen a la solución de problemas de salud de la sociedad, la solución de problemas ambientales y a la ejecución de proyectos de investigación que contribuyen a mejorar la calidad de vida del ser humano.

Impacto de la investigación. Son los efectos logrados por los resultados de la investigación en lo académico, social, ambiental, económico, científico, tecnológico, político, cultural, entre otros, en corto, mediano y largo plazo; de los cuales la universidad tiene evidencias y reconocimiento en su entorno de esos impactos. Ese valuado desde los impactos sociales tangibles por proyectos de investigación ejecutados por la universidad, por el aprovechamiento de los impactos por la universidad de sus investigaciones para mejorar lo académico e impactos económicos percibidos por la investigación desarrollada en la universidad.

Capital negocio. Se refiere a todas las relaciones establecidas por la universidad con agentes externos con el fin de generar investigación. Los activos intangibles que lo conforman son:

Relaciones con otras universidades para desarrollar investigación. Son todas las relaciones establecidas por medio de convenios formales entre dos o más universidades con el fin de desarrollar investigaciones que le permitan generar capital intelectual. Es valorado por la existencia de convenios firmados con otras universidades, proyectos de investigación ejecutados en alianzas con investigadores de otras universidades y la producción científica generada a partir de investigaciones con otras universidades. La tabla 3 muestra los indicadores para medir el capital relacional. 
Tabla 3

Indicadores para medir el capital relacional Indicador

Número de investigadores con apoyo económico recibido para participar en eventos internacionales/ Número de investigadores

Número de eventos internos organizados para dar a conocer productos de investigación con apoyo económico por la universidad

Número de investigadores participantes en asociaciones externas de tipo científico

Número de personas externas a la universidad beneficiadas por la ejecución de proyectos de investigación en el área de la salud

Número de personas externas a la universidad beneficiadas por la ejecución de proyectos de investigación en el área ambiental

Número de personas con calidad de vida mejorada por proyectos de investigación ejecutados por la universidad

Número de impactos sociales tangibles obtenidos por proyectos de investigación

Número de cursos académicos mejorados por resultados de proyectos de investigación

Valor en bienes tangibles obtenidos por proyectos de investigación / Valor total de bienes destinados a la investigación

Número de convenios firmados con otras universidades para desarrollar investigación/ Número de convenios totales establecidos por la universidad

Número de proyectos de investigación ejecutados en alianzas con investigadores de otras universidades/ Número total de proyectos de investigación ejecutados

Número de productos científicos generados a partir de investigaciones con otras universidades/ Número de productos científicos generados

Elaboración propia (2014). Producto del análisis de la información desde los resultados de la encuesta realizada a investigadores en las universidades públicas de la Costa Caribe de Colombia.

\subsubsection{Capital digital}

Conjunto de recursos intangibles colocados a disposición de sus usuarios por medio de la utilización del internet, las actividades de transmisión de datos, documentos digitales, los contenidos multimedia, los programas software, o el intercambio de productos y servicios en línea, entre otros. Los activos intangibles que lo conforman son:

Repositorios virtuales. Es un almacén virtual de documentos de diversos formatos y procedencia, ordenados por características comunes; tienen el propósito de almacenar, preservar y difundir información digital, accesibles desde internet o una intranet. Son instrumentos del autoarchivo de acceso abierto, capaces de reunir la producción intelectual de una disciplina o de una institución. Es evaluado por permitir consultar información sobre la investigación y sus productos desde el exterior de la universidad, disponibilidad de bases de datos científicas para desarrollar investigación y la disponibilidad de libros digitales incluidos en la biblioteca de la universidad como material de consulta desde cualquier sitio. La tabla 4 muestra los indicadores para medir el capital digital. 
Tabla 4

Indicadores para medir el capital digital

Indicador

Número de consultas externas realizadas a la página web de investigación/Número de consultas totales

Número de consultas a bases de datos científicas de uso en la universidad/Número de consultas totales

Número de consultas externas a libros digitales incluidos en la biblioteca de la universidad/Número de consultas totales

Número de consultas internas a libros digitales incluidos en la biblioteca de la universidad/Número de consultas totales

Fuente: Elaboración propia (2014). Producto del análisis de la información desde los resultados de la encuesta realizada a investigadores en las universidades públicas de la Costa Caribe de Colombia.

\subsubsection{Características del modelo.}

El grafico 3 permite observar la agrupación de los activos intangibles de las universidades de la Costa Caribe colombiana acorde con su naturaleza en cuatro componentes principales: el capital humano; el capital digital; el capital relacional formado por el capital social y el capital negocio; el capital estructural formado por el capital organizativo y el capital tecnológico.

En ese orden, el modelo propuesto considera treinta y cuatro variables o tipos de activos intangibles agrupados en once elementos o grupos de activos intangibles que forman parte de los cuatro componentes del capital intelectual de las universidades de la Costa Caribe colombiana, integrando cada componente mencionado anteriormente. 


\section{Grafico 3}

Modelo de capital intelectual para la Investigación en las universidades públicas de la Costa Caribe colombiana.

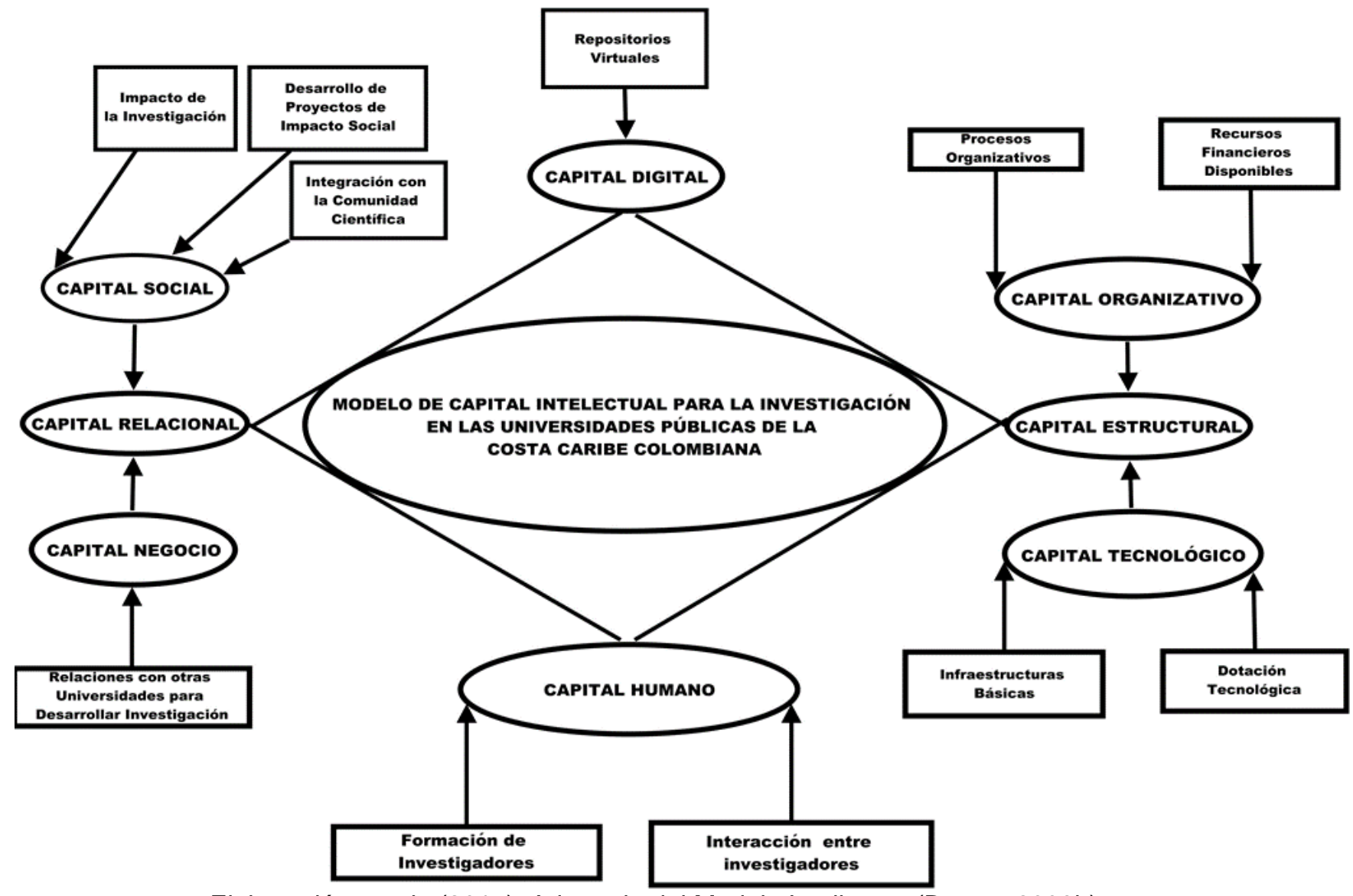

Elaboración propia (2015). Adaptado del Modelo Intellectus (Bueno, 2003b).

Como se puede ver, el modelo posee una estructura interrelacionada y abierta producto de los agentes o entes de conocimiento: los investigadores de las universidades públicas de la Costa Caribe colombiana acorde con la realidad donde se desenvuelven. La fortaleza interna del modelo se fundamenta en la dinámica del capital humano basado en el capital estructural, teniendo en cuenta el capital organizativo y el capital tecnológico; así mismo, la fortaleza externa se fundamenta en el capital relacional por medio de la dinámica del capital social y el capital negocio. El capital digital permite la interconexión de los componentes anteriores y le agrega ventaja competitiva basada en la expresión y visualización de cada uno de sus productos. 


\section{Conclusiones}

Para finalizar, en correspondencia con los resultados analizados para cada objetivo planteado, es preciso afirmar que los componentes capital humano, capital estructural y capital digital son utilizados de forma favorable para la investigación en las universidades públicas de la Costa Caribe colombiana, mientras que el capital relacional tiene una presencia desfavorable para el desarrollo de la investigación con el fin de generar capital intelectual.

Igualmente, existe en las universidades de la Costa Caribe colombiana una presencia favorable de activos intangibles pertenecientes al capital social, al capital estructural y al capital relacional tales como, la integración con la comunidad científica, el desarrollo de proyectos de impacto social, los procesos organizativos, las infraestructuras básicas para la investigación y los impactos de la investigación.

Cabe destacar, la presencia desfavorable de activos intangibles como la existencia de un sistema de categorización de investigadores, docentes dedicados exclusivamente a investigar, empresarios vinculados a la universidad como investigadores, incentivos a la investigación diferente al salarial por producción científica, convenios firmados con empresas de los sectores económicos para desarrollar investigación, la utilización de redes de conocimiento para generar capital intelectual desde la investigación y la existencia en la universidad de un portafolio de productos y servicios dirigido a la comercialización del conocimiento producido por la investigación.

Acorde con lo anterior, el modelo propuesto permite dinamizar cada uno de sus componentes principales, capital humano, capital estructural y capital relacional, incluye como aporte de este estudio el componente capital digital. Para cada uno se definen sus elementos o activos intangibles, sus indicadores o forma cómo se pueden medir y evaluar en cada institución; considera treinta y cuatro variables o tipos de activos intangibles agrupados en once elementos o grupos de activos intangibles que forman parte de los cuatro componentes del capital intelectual de las universidades de la Costa Caribe colombiana.

Además, se hace necesario adaptar el modelo propuesto de capital intelectual para la investigación en universidades públicas de la Costa Caribe colombiana como estrategia de orientación para determinar y valorar el capital intelectual y los activos intangibles producidos desde la investigación identificada con las características de cada universidad. Como también, fortalecer sus procesos de autoevaluación con el fin de determinar el capital intelectual que poseen para visibilizarlo, obtener provecho de sus ventajas competitivas y 
valores agregados como factores diferenciales que la distingan de otras instituciones de educación superior.

De igual manera se recomienda formular una línea de investigación en capital intelectual vinculada a los programas de postgrados de investigación ofertados por la universidad con el fin de profundizar en esta temática de suma importancia para la universidad.

\section{Referencias}

Bueno Campo, Eduardo, Salmador, María Paz y Merino, Carlos. (2008). Génesis, concepto y desarrollo del capital intelectual en la economía del conocimiento. Una reflexión sobre el Modelo Intellectus y sus aplicaciones. Estudios de Economía Aplicada, 26(2), 43-63. Recuperado de http://www.redalyc.org/articulo.oa?id=30113187003

Bueno Campo, Eduardo. (1999). La economía del conocimiento: la importancia de los intangibles. San Lorenzo del Escorial, España: IU Euroforum Escorial.

Bueno Campo, Eduardo. (2003a). Gestión del Conocimiento en Universidades y Organismos Públicos de Investigación. Madrid, España: Universidad Autónoma de Madrid. Dirección General de Investigación, Consejería de Educación.

Bueno Campo, Eduardo. (2003b). Modelo Intellectus: Medición y Gestión del Capital Intelectual (Documentos Intellectus $\mathrm{N}^{\circ}$ 5). Madrid: IADE. Universidad Autónoma de Madrid, España.

Bustos Farías, Eduardo. (2008). La influencia del capital intelectual en la gestión de calidad en instituciones de educación superior: el caso de la Escuela Superior de Cómputo del Instituto Politécnico Nacional México (Tesis para optar el grado de Doctorado en Ciencias Administrativas). Instituto Politécnico Nacional, México.

Carrillo, Francisco Javier. (2000). Gestión del conocimiento. Instituto Tecnológico de Monterrey. Gestión del conocimiento y capital intelectual (Colección Cuadernos de Trabajo -formación, empleo, cualificaciones, №31). San Sebastián, España: Centro de Investigación y Documentación sobre problemas de la Economía, el Empleo y las Cualificaciones Profesionales.

Cobo Jiménez, Antonio. (2006). Modelo de capital intelectual de la Consejería de Educación de la Junta de Andalucía. Indicadores de capital humano y gestión del conocimiento (Tesis para optar el grado de Doctorado de Economía y Administración de Empresas), Universidad de Málaga, España.

Colciencias, Departamento Administrativo de Ciencia, Tecnología e Innovación. (2013). Manual del aplicativo GrupLAC. Directorio de Grupos de Investigación. Bogotá, Colombia: Colciencias.

Colciencias. (2012). InstituLac manual de usuarios. Bogotá, Colombia: Colciencias.

Colciencias. (2014). Manual de usuario CvLac. Bogotá, Colombia: Colciencias. 
Colombia, Congreso. (08 de febrero, 1994). Ley 115. Por la cual se expide la ley general de educación. Bogotá: Congreso de Colombia. Ministerio de Educación Nacional de Colombia.

Colombia, Congreso. (19 de Julio, 2002). Ley 749. Por la cual se organiza el servicio público de la educación superior en las modalidades de formación técnica profesional y tecnológica, y se dictan otras disposiciones. Bogotá: Congreso de Colombia. Ministerio de Educación Nacional de Colombia.

Colombia, Congreso. (23 de enero, 2009). Ley 1286. Por la cual se modifica la Ley 29 de 1990, se transforma a Colciencias en Departamento Administrativo, se fortalece el Sistema Nacional de Ciencia, Tecnología e Innovación en Colombia y se dictan otras disposiciones. Bogotá: Congreso de Colombia.

Colombia, Congreso. (25 de abril, 2008). Ley 1188. Por la cual se regula el registro calificado de programas de educación superior y se dictan otras disposiciones. Bogotá: Congreso de Colombia. Ministerio de Educación Nacional de Colombia.

Colombia, Congreso. (30 de diciembre, 1992). Ley 30. Por la cual se organiza el servicio público de la Educación Superior. Bogotá: Congreso de Colombia. Ministerio de Educación Nacional de Colombia.

Colombia. (20 de abril, 2010). Decreto 1295. Por el cual se reglamenta el registro calificado de que trata la Ley 1188 de 2008 y la oferta y desarrollo de programas académicos de educación superior. Bogotá: Ministerio de Educación Nacional de Colombia.

Consejo Nacional de Acreditación -CNA. (2009). Lineamientos para la Acreditación de Alta Calidad de Programas de Maestría y Doctorado. Bogotá, Colombia: CNA.

Da Silva Santos Rodrigues, Helena Maria, Figueroa Dorrego, Pedro Froilán y FernándezJardón Fernández, Carlos María. (2009). La influencia del capital intelectual en la capacidad de innovación de las empresas del sector de automoción de la eurorregión galicia norte de portugal. Recuperado de https://www.academia.edu/1191687/La influencia del capital intelectual en la capac idad de innovaci\%C3\%B3n de las empresas del sector de automoci\%C3\%B3n d e la eurorregi\%C3\%B3n Galizia Norte de Portugal?auto=download

Farfán Buitrago, Dalsy Yolima y Garzón Castrillón, Manuel Alfonso. (2006). La gestión del conocimiento. Colombia: Editorial Universidad del Rosario.

Fazlagic, Amir. (2005). Measuring the capital intellectual of a university. Paper presented at the Conference on Trends in the Management of Human Resources in Higher Education. Recuperado de https://www.oecd.org/dataoecd/56/16/35322785.pdf

Funes Cataño, Yolanda. (2007). Valuación de los activos intangibles. El caso de la UNAM (Tesis Doctoral en Ciencias de la Administración). Facultad de Contaduría y Administración, Universidad Nacional Autónoma de México, México.

Hazelkorn, Ellen. (2005). University Research Management. Developing research in new institutions. París, France: OECD. 
Hernández Valencia, Ramón Albeiro, Cardona Orozco, Gloria Marcelia, Cardona Henao, José Fernando y Lasso Ramírez, Norma Constanza. (2007). Estado actual del capital intelectual y la gestión del conocimiento en las universidades del Risaralda. Memorias. Revista de Investigaciones Universidad Cooperativa de Colombia, 3(10), 49-65. Recuperado de http://revistas.ucc.edu.co/index.php/me/article/view/265/262

Leal de Suárez, Raíza Beatriz. (2003). Sistema de indicadores para evaluar la gestión del capital intelectual en las organizaciones universitarias. Universitas 2000, 25(1-2), 127144.

Leitner, Karl-Heinz (2004). Intellectual capital reporting for universities: conceptual background and application for Austrian universities. Research Evaluation, 13(2), 129140.

Molina López, Evaristo (2001). Construcción de indicadores y parámetros. Revista Latinoamericana de Estudios Educativos, 31(002), 119-123.

Molina Parra, Paula Andrea, Arango Serna, Martín Darío y Botero Botero, Sergio. (2010). Análisis del valor agregado del conocimiento: caso aplicado en una institución de educación superior. Revista Facultad de Ciencias Económicas, 18(2). Recuperado de: http://www.scielo.org.co/scielo.php?script=sci arttext\&pid=S0121-68052010000200006

Mosquera, Cardona, Luz Enith. (2011). Gestión del capital intelectual de las instituciones educación superior. Caso Universidad Nacional de Colombia. (Trabajo final presentado como requisito parcial para optar al título de Magister en Administración). Facultad de Administración, Sede Manizales, Universidad Nacional de Colombia, Colombia.

Rivas Crisóstomo, Ignacio. (2004). Contribuciones de la Administración Estratégica al desarrollo de los estudios de posgrado en el Instituto Politécnico Nacional (Tesis para obtener el grado de la maestría en Ciencias con especialidad en Negocios). Instituto Politécnico Nacional, Escuela Superior de Comercio y Administración, Unidad Santo Tomás, México.

Sánchez, Paloma, Castrillo, Rocío y Elena, Susana. (2006). Intellectual capital management and reporting in universities. Usefulness, comparability and diffusion, best practices in data gathering and analysis from the Autonomous University of Madrid's experience. Recuperado de http://citeseerx.ist.psu.edu/viewdoc/summary?doi=10.1.1.464.4705

Seguí Mas, Elies. (2007). La gestión del capital intelectual en las entidades financieras. Caracterización del capital humano en las cooperativas de crédito (Tesis doctoral). Departament d'Economia i CCSS - Universitat Politécnica de Valencia, Valencia, España.

Tapscott, Don, Ticoll, David y Lowy, Alex. (2000). Capital digital. El poder de las redes de negocios (Trad. M. Condor). España: Taurus digital.

Tapscott, Don. (2000). La creación de valor en la economía digital [Creating Value in the Network Economy, Guillermo Masio]. Argentina: Ediciones Granica S.A.

Villaveces Cardoso, José Luis. (2010). La investigación en Uniandes: construcción de una política. Bogotá: Universidad de los Andes, Vicerrectoría de Investigaciones, Ediciones Uniandes. 